\title{
CLASSIFICATION AND DIAGNOSIS OF SCHIZOPHRENIA OR OTHER PRIMARY PSYCHOTIC DISORDERS: CHANGES FROM ICD-10 TO ICD-11 AND IMPLEMENTATION IN CLINICAL PRACTICE
}

\author{
Wolfgang Gaebel ${ }^{1,2}$, Ariane Kerst ${ }^{1,2}$ \& Johannes Stricker ${ }^{1,2}$ \\ ${ }^{1}$ Department of Psychiatry and Psychotherapy, Medical Faculty, LVR-Klinikum Düsseldorf, Heinrich-Heine-University, \\ Düsseldorf, Germany \\ ${ }^{2}$ WHO Collaborating Centre on Quality Assurance and Empowerment in Mental Health, Düsseldorf, Germany
}

received: 15.4.2020;

revised: 5.5.2020;

accepted: 23.5.2020

\begin{abstract}
SUMMARY
From January 2022, the WHO member countries shall start implementing the mortality and morbidity statistics (MMS) version of the eleventh revision of the International Classification of Diseases (ICD-11). Regarding mental, behavioural or neurodevelopmental disorders, there are substantial changes from ICD-10 to ICD-11. The subchapter for schizophrenia or other primary psychotic disorders has changed due to a revised structure, new diagnostic criteria, and the introduction of dimensional elements (i.e., course and symptom qualifiers). The aim of this manuscript is twofold. First, we review changes from ICD-10 to ICD11 in the classification and diagnosis of schizophrenia or other primary psychotic disorders, including findings from recent field studies. Second, we provide an overview of approaches to the implementation of ICD-11 in clinical practice. Critical elements for transition from ICD-10 to ICD-11 include the use of digital tools, education and training, stakeholder involvement, national adaptations, and continuous evaluation.
\end{abstract}

Key words: ICD-11 - International Classification of Diseases - Schizophrenia or Other Primary Psychotic Disorders - implementation

\section{INTRODUCTION}

In May 2019, the World Health Assembly (WHA) endorsed the eleventh revision of the International Classification of Diseases (ICD-11). Starting in 2022, World Health Organization (WHO) member countries will implement ICD-11. To meet the needs of different user groups, different versions of the ICD-11 will be available. In addition to the Mortality and Morbidity Statistics (MMS) version, also more detailed Clinical Descriptions and Diagnostic Guidelines (CDDG) for the mental, behavioural or neurodevelopmental disorders chapter (MBND) are currently under development. The CDDG provide detailed descriptions and guidance on the diagnosis of mental disorders including information related to the boundaries with other disorders (differential diagnosis) and with normality. Additionally, a primary care version of the MBND chapter is under development and a research version will possibly be available at a later stage of the implementation phase.

In an extensive international revision process, followed by worldwide field trials, the classification has undergone several changes, following the guiding principles of global applicability, scientific validity, and clinical utility. ICD-11 allows for more extensive and flexible coding compared to ICD-10 and has taken steps towards dimensional assessments. The MBND chapter has been restructured and now includes six subchapters more than ICD-10. Existing subchapters were revised and new diagnostic categories were added. Additionally,
ICD-11 adopts a lifespan-approach. Thus, mental disorders that primarily occur in childhood and adolescence are not grouped into a separate disorder grouping but are integrated into other disorder groupings according to their symptomatology. Moreover, ICD-11 provides guidance for classification adjustments to specific cultural contexts (Gureje et al. 2020). For more a detailed overview of changes from ICD-10 to ICD-11 for the MBND chapter see Gaebel et al. 2020, Reed et al. 2019, or Stein et al 2020. In the following paragraphs, we will focus on changes and innovations in the Schizophrenia and Other Primary Psychotic Disorders subchapter. Additionally, we will discuss the implementation of the ICD-11 MBND chapter in clinical practice.

\section{ICD-11 SCHIZOPHRENIA OR OTHER PRIMARY PSYCHOTIC DISORDERS}

The ICD-11 Schizophrenia or Other Primary Psychotic Disorders subchapter replaces the ICD-10 Schizophrenia, Schizotypal and Delusional Disorders subchapter. ICD-11 Schizophrenia or Other Primary Psychotic Disorders are characterized by "significant impairments in reality testing and alterations in behaviour manifest in positive symptoms such as persistent delusions, persistent hallucinations, disorganized thinking (typically manifest as disorganized speech), grossly disorganized behaviour, and experiences of passivity and control, negative symptoms such as blunted or flat affect and avolition, and psychomotor disturbances” (WHO 2019a). 
Table 1. Brief Descriptions of Diagnostic Categories in the ICD-11 Schizophrenia or Other Primary Psychotic Disorders Subchapter (adopted from WHO 2019a)

\begin{tabular}{|c|c|}
\hline Diagnostic Category & Brief Description \\
\hline Schizophrenia & $\begin{array}{l}\text { Characterized by disturbances in multiple mental modalities, including thinking (e.g., } \\
\text { delusions, disorganization in the form of thought), perception (e.g., hallucinations), self- } \\
\text { experience (e.g., the experience that one's thoughts or behaviour are under the control of an } \\
\text { external force), cognition (e.g., impaired attention), volition (e.g., loss of motivation), affect } \\
\text { (e.g., blunted emotional expression), and behaviour (e.g., bizarre behaviour). Symptoms } \\
\text { must be present for at least one month. }\end{array}$ \\
\hline Schizoaffective disorder & $\begin{array}{l}\text { Episodic disorder in which the diagnostic requirements of schizophrenia and a manic, } \\
\text { mixed, or moderate or severe depressive episode are met within the same episode of illness. }\end{array}$ \\
\hline Schizotypal disorder & $\begin{array}{l}\text { Characterized by an enduring pattern (i.e., at least several years) of eccentricities in } \\
\text { behaviour, appearance and speech, accompanied by cognitive and perceptual distortions, } \\
\text { unusual beliefs, and discomfort with interpersonal relationships. }\end{array}$ \\
\hline $\begin{array}{l}\text { Acute and transient } \\
\text { psychotic disorder (ATPD) }\end{array}$ & $\begin{array}{l}\text { Characterized by an acute onset of psychotic symptoms that emerge without a prodrome and } \\
\text { reach their maximal severity within two weeks. }\end{array}$ \\
\hline Delusional disorder & $\begin{array}{l}\text { Characterized by the development of a delusion or a set of related delusions that persist for } \\
\text { at least three months (usually much longer), which occur in the absence of a depressive, ma- } \\
\text { nic, or mixed mood episode. Other characteristic symptoms of schizophrenia are not present. }\end{array}$ \\
\hline
\end{tabular}

Non-primary psychotic disorders, e.g. due to substance use, are placed in the subchapter for the respective mental disorder or medical condition (e.g., cocaineinduced psychotic disorder in the Disorders due to Substance Abuse or Addictive Behaviours subchapter). Table 1 displays an overview of the diagnostic categories in the ICD-11 Schizophrenia or Other Primary Psychotic Disorders subchapter.

For all primary psychotic disorders, dimensional course- and symptom qualifiers are introduced in ICD11. These qualifiers allow a more precise and individual description of illness presentation and course compared to ICD-10 (Gaebel \& Kerst 2019, Gaebel et al. 2020). Dimensional symptom qualifiers allow for specification of the current symptomatology in six symptom domains (positive symptoms, negative symptoms, depressive mood symptoms, manic mood symptoms, psychomotor symptoms, cognitive symptoms) on a 4-point scale ranging from $0=$ not present to $3=$ present and severe. The positive symptom domain includes hallucinations, delusions, disorganized thinking, disorganized behaviour, and experiences of passivity and control. The negative symptom domain includes alogia, limited affect, avolition, anhedonia, and asociality. The depressive mood symptoms domain includes depressed mood and related symptoms. The manic mood symptoms domain includes elevated, irritable or expansive mood and increase in energy. The psychomotor symptoms domain includes psychomotor agitation, psychomotor retardation, and catatonic symptoms. Finally, the cognitive symptoms domain includes deficits in speed of processing, attention/concentration, orientation, judgment, abstraction, verbal or visual learning, and working memory.

Course qualifiers allow for specification of the longitudinal course and current cross-sectional status of primary psychotic disorders. Regarding longitudinal course, the course specifiers differentiate between the first episode, multiple episodes (i.e., minimum of two episodes with at least three months of partial or full remission), and continuous course (i.e., symptoms present for almost all of the course for at least one year). Regarding cross-sectional status, the course qualifiers allow for a differentiation of the current symptomatology as currently symptomatic, in partial remission or in full remission.

Major changes in the diagnostic categories pertain to schizophrenia and ATPD. In ICD-11, schizophrenia subtypes are omitted in favour of a more detailed characterization of the schizophrenia diagnosis by means of course- and symptom-qualifiers. This change reflects the evidence indicating the longitudinal instability and prognostic invalidity of schizophrenia subtypes (Gaebel 2012, Gaebel et al. 2013, 2015). Schizophrenia symptom characteristics and the duration criterion in ICD-11 have largely remained the same compared to ICD-10, although first rank symptoms have been deemphasized (see WHO 2019a for more details). Core symptoms of schizophrenia include persistent delusions, persistent hallucinations, thought disorder, and experiences of influence, passivity, or control (present for a least one month).

Figure 1 depicts changes in ATPD from ICD-10 to ICD-11. ICD-10 included four subtypes of ATPD. ICD10 Acute Polymorphic Psychotic Disorder Without Symptoms of Schizophrenia largely corresponds to ICD-11 Acute and Transient Psychotic Disorder. ICD10 Acute Polymorphic Psychotic Disorder With Symptoms of Schizophrenia and ICD-10 Acute Schizophrenia-like Psychotic Disorder account for presentations of schizophrenia-like symptoms that are too short to qualify for a diagnosis of schizophrenia, and are thus included in the diagnosis of Other Primary Psychotic Disorder in ICD-11. ICD-10 Other Acute Predominantly Delusional Psychotic Disorder together with ICD-10 


\section{ICD-10}

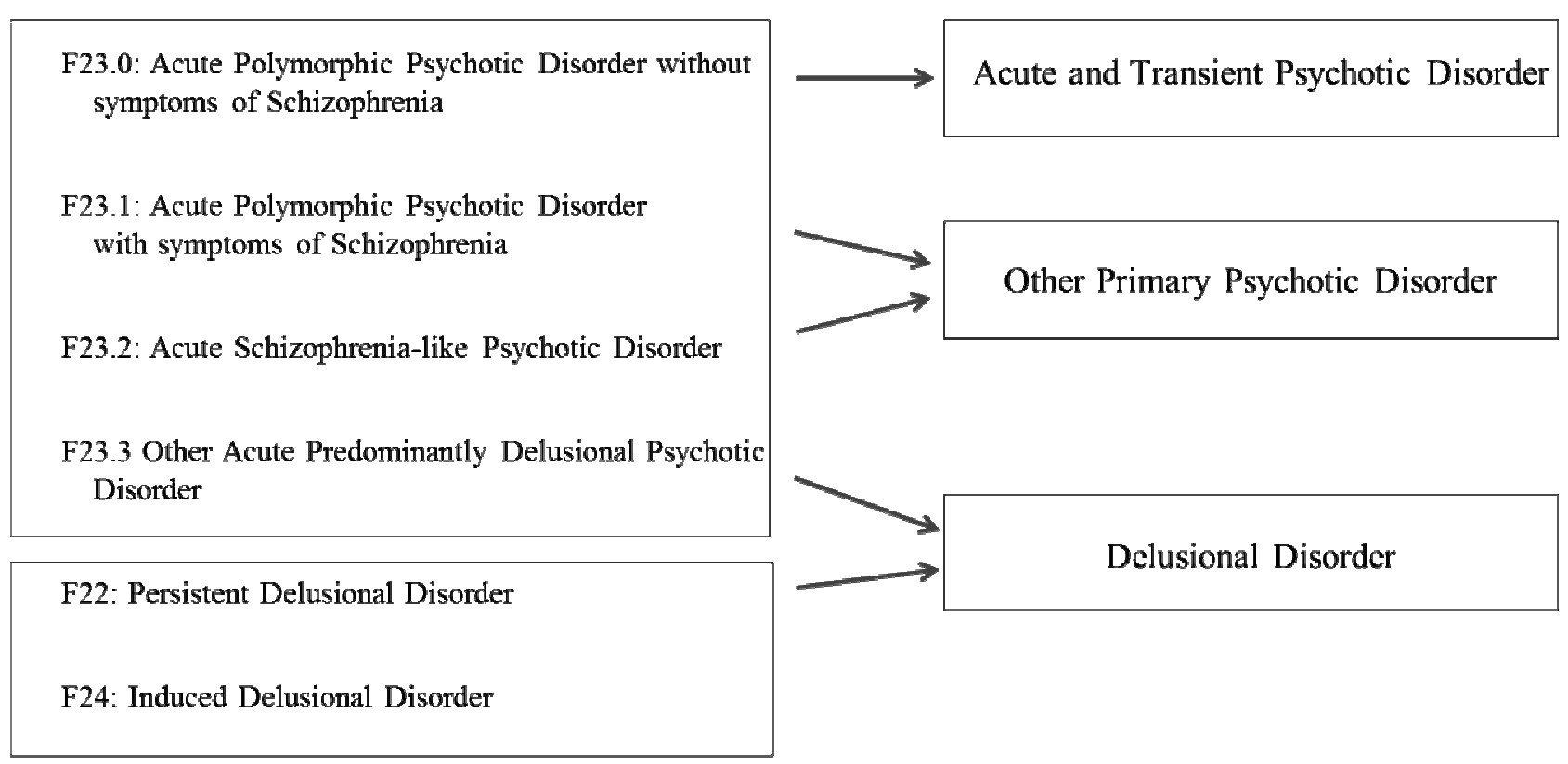

Figure 1. Changes from ICD-10 to ICD-11 in ATPD and delusional disorder

Persistent Delusional Disorder correspond to ICD-11 Delusional Disorder. In ICD 11, ATPD is characterized by an acute onset of psychotic symptoms that emerge without a prodrome, reach their maximal severity within two weeks and persist for no more than three months. Positive psychotic symptoms in ATPD may fluctuate rapidly and vary in their intensity over a short period of time. The ICD-10 ATPD subtypes were eliminated due to limited empirical evidence and in favour of clinical utility (Stein et al. 2020).

Schizoaffective disorder has an episodic illness course. Persons with a schizoaffective disorder fulfil the criteria for a diagnosis of schizophrenia and simultaneously the criteria for a manic, mixed, moderate or severe depressive episode (WHO 2019a). Thus, in schizoaffective disorder, prominent symptoms of schizophrenia and affective episodes are combined. The symptoms are either present at the same time or separated by a few days and need to persist for at least one month (WHO 2019a). Studies show that the majority of patients with schizoaffective disorder have more than one episode. Episodes of schizoaffective disorder may even occur decades apart from each other (Stein et al. 2020, Marneros 2003).

Schizotypal disorder is defined as an enduring pattern of unusual speech, perceptions, beliefs, and behaviours of insufficient intensity to meet requirements for another psychotic disorder in ICD-10 and in ICD11. Yet, schizotypal disorder may be a possible predecessor of schizophrenia (Jablensky 2011, Stein et al. 2020) and is therefore kept in the ICD-11 chapter of primary psychotic disorders, contrary to DSM-5 which classifies schizotypal disorder as a personality disorder.
To increase clarity, simplicity, and clinical utility, ICD-11 collapses three ICD-10 diagnostic categories into one ICD-11 delusional disorder diagnostic category (i.e., Other Acute Predominantly Delusional Disorder, Persistent Delusional Disorder, and Induced Delusional Disorder; see Figure 1). According to ICD-11, delusional disorder usually persists for more than three months and is characterized by the presence of a delusion or set of related delusions that is relatively stable and fixed (WHO 2019a). Other psychotic symptoms are absent, although some specific hallucinations related to the delusional theme may occur. Additionally, affect, speech, and behaviour are usually unaffected outside delusional themes.

Recent evaluative field studies provide evidence for the reliability and perceived clinical utility of the diagnostic categories in the ICD-11 Schizophrenia or Other Primary Psychotic Disorders subchapter. For example, a large clinical field study with 28 participating centres in 13 countries found a higher reliability for schizophrenia, schizoaffective disorder, ATPD, and delusional disorder in ICD-11 compared to previous reliability estimates for ICD-10 (Reed et al. 2018). Similarly, a clinical field study with 23 Mexican clinicians and 153 patients reported strong diagnostic reliability for schizophrenia or other primary psychotic disorders (Kappa $=0.83$; Medina-Mora et al. 2019). In a vignette-based field study with 928 health professionals from all WHO regions, the ICD-11 diagnostic guidelines for schizoaffective disorder were perceived as clearer and easier to use compared to the ICD-10 diagnostic guidelines for schizoaffective disorder (Gaebel et al. 2019). In this specific study, no statistically significant differences between ICD-11 and ICD-10 in diagnostic accuracy for schizophrenia and schizoaffective disorder were observed. 


\section{ICD-11 IMPLEMENTATION}

The revision and implementation of the ICD-11 follows a structured project planning process which foresees the use of the revised classification in WHO member countries from the beginning of 2022. The WHO implementation plan covers different thematic areas that are central for a successful transition from ICD-10 to ICD-11 across different global regions (WHO 2019b). The WHO translates ICD-11 into the six official United Nations languages (English, French, Spanish, Russian, Chinese, and Arabic). Translations to further languages are conducted by the respective countries. In the implementation process, the translations of the classification, supporting materials, and training courses need to be completed first. The translation process focuses on the representation of equivalent concepts of the classification rather than a wordby-word translation. This approach also allows for adjustments to diverse cultural contexts. Computerized tools and experts for language interpretation support the translation (Gaebel \& Kerst 2019).

Another important thematic area of the implementation plan is capacity building, which includes the evaluation of existing coding capacities of the WHO member countries, the development of training tools, and the provision of specialist training adapted to identified needs. In collaboration with national stakeholders, WHO is evaluating the impact of those training activities and finally plans the development of a coder profile as well as coder certification for ICD-11 (WHO, 2019b). According to the ICD-11 field trials, the training of professionals in diagnosing and coding of MBND may be one of the key factors for successful transition to ICD-11 (Gaebel et al. 2018). Thus, an integrated setting- and workforce-adapted implementation with guided education and training plays a major role in the implementation process. So far, training courses on the use of ICD-11 including CDDG and vignette-based practice sessions have already been conducted at various international scientific meetings. Currently, WHO together with the Columbia University Global Mental Health Programme is developing online training modules for ICD-11 that cover diagnostic guidelines, essential changes from ICD-10, and multimedia case vignettes with feedback for training purposes. These training modules will be available in a WHO online program called Global Mental Health Academy (GCPN 2019).

Information technology infrastructure is another priority area of the ICD-11 structured implementation. This topic includes the technology needs-assessment in member countries and the promotion of freely-available computer tools and expert staff. National information systems need to be integrated into the transition process and updated, if needed, to guarantee functionality and interoperability of systems. In addition to the national contexts, the quality of data and their international comparability need to be ensured. Following implementation, the impact of the switch from ICD-10 to ICD-11 on health data (e.g., prevalence estimates) will be analysed, including reimbursement schemes, mortality and morbidity statistics, and legal frameworks. The quality of coding will be evaluated on a caseselection basis and changes arising from the transition to ICD-11 will be monitored. To cover those mentioned priority areas adequately, WHO provides a freely-available ICD-11 implementation package, which includes the ICD-11 Implementation or Transition Guide (WHO 2019b), the ICD-11 Coding Tool, the ICD-11 browser for Mortality and Morbidity Statistics, the ICD-11 Reference Guide and the ICD-10/ICD-11 Mapping Tables (WHO 2019a).

The Implementation or Transition Guide summarizes essential issues that member countries need to consider in the transition from ICD-10 to ICD-11 (WHO 2019b). Rather than considering complex local circumstances, the document provides general guidelines for decision makers and gives recommendations for key actions related to the priority areas mentioned above (e.g., the establishment of a national centre of excellence, system maintenance or project management). The ICD-11 Browser and the Coding Tool may be used to increase familiarity with the ICD-11 structure and coding procedure. The Reference Guide provides thorough background information related to ICD-11 and its use. In summary, those tools provide a solid basis to facilitate the implementation of ICD-11. Additionally, stakeholder involvement and adaptation to local circumstances are key to successfully transition from ICD-10 to ICD-11.

\section{CONCLUSION}

The ICD-11 was developed in an international multidisciplinary and multi-stakeholder revision process followed by extensive evaluative field-testing. The major aims of the revision process were applicability, scientific reliability and validity, and clinical utility. The ICD-11 Schizophrenia or other Primary Psychotic Disorders subchapter adopts dimensional elements of psychiatric classification in the form of symptom - and course qualifiers. The chapter has been restructured and partly simplified for improved clinical utility. Fieldstudies have shown advantages of the ICD-11 compared to ICD-10 regarding the classification of primary psychotic disorders. The international implementation of the ICD-11 needs to follow a well-structured strategical and methodological plan. Involvement of national stakeholders and national adaptations are required to assure a successful transition from ICD-10 to ICD11. Focus areas include translation, specialist training and education, capacity building and adaptation of technical infrastructure. 


\section{Acknowledgements:}

Disclosure: Wolfgang Gaebel was a member of the WHO TAG on MBND, and chair of the WHO ICD-11 working group on Schizophrenia and Other Primary Psychotic Disorders. He is a member of the WHO Field Study Coordination Group, of the WHO International Advisory Group for Training and Implementation for ICD-11 MBND, and of the WHO FIC Medical and Scientific Advisory Committee.

Conflict of interest : None to declare.

\section{Contribution of individual authors:}

Wolfgang Gaebel conceptualized the manuscript. Wolfgang Gaebel, Ariane Kerst \& Johannes Stricker wrote the manuscript.

\section{References}

1. Gaebel W, Kerst A: ICD-11 Mental, behavioural or neurodevelopmental disorders: innovations and managing implementation. Archives of Psychiatry and Psychotherapy 2019; 3:7-12

2. Gaebel W, Stricker J \& Kerst A: Changes from ICD-10 to ICD-11 and future directions in psychiatric classification. Dialogues Clin Neurosci 2020; 22:7-15

3. Gaebel W, Stricker J, Riesbeck M, Zielasek J, Kerst A, Meisenzahl-Lechner $E$ et al.: Accuracy of diagnostic classification and clinical utility assessment of ICD-11 compared to ICD-10 in 10 mental disorders: findings from a web-based field study. Eur Arch Psychiatry Clin Neurosci 2019; 270:81-89

4. Gaebel W, Zielasek J \& Reed GM: Mental and behavioural disorders in the ICD-11: Concepts, methodologies, and current status. Psychiatr Pol 2017; 51:169-95

5. Gaebel W: Status of psychotic disorders in ICD-11. Schizophr Bull 2012; 38:895-98
6. Gaebel W, Zielasek J \& Cleveland HR: Psychotic disorders in ICD-11. Die Psychiatrie; 2013; 10:11-17

7. Gaebel W, Zielasek $J$ \& Falkai P: Psychotic disorders in ICD-11. Die Psychiatrie 2015; 12:71-76

8. Global Clinical Practice Network (GCPN): The ICD-11 is here. 2019. Available at https://gcp.network/en/globalmental-health-news-and-events/single/icd-11-adopted

9. Gureje O, Lewis-Fernandez $R$, Hall BJ \& Reed GM: Cultural considerations in the classification of mental disorders: Why and how in ICD-11. BMC Medicine 2020; 18:1-2

10. Jablensky A: Diagnosis and revision of the classification system. In Gaebel W (ed.): Schizophrenia: Current science and clinical practice. 1-30. Wiley, 2011

11. Marneros A. The schizoaffective phenomenon: The state of the art. Acta Psychiatr Scand 2003; 108:29-33

12. Medina-Mora ME, Robles R, Rebello TJ, Domínguez T, Martínez $N$, Juárez $F$ et al.: ICD-11 guidelines for psychotic, mood, anxiety and stress-related disorders in Mexico: Clinical utility and reliability. Int J Clin Health Psychol 2019; 19:1-11

13. Reed GM, Sharan P, Rebello TJ, Keeley JW, MedinaMora ME, Gureje $O$ et al.: The ICD 11 developmental field study of reliability of diagnoses of high-burden mental disorders: Results among adult patients in mental health settings of 13 countries. World Psychiatry 2018; 17:174-86

14. Reed GM, First MB, Kogan CS, Hyman SE, Gureje O, Gaebel $W$ et al.: Innovations and changes in the ICD-11 classification of mental, behavioural and neurodevelopmental disorders. World Psychiatry 2019; 18:3-19

15. Stein DJ, Szatmari P, Gaebel W, Berk M, Vieta E, Maj M et al.: Mental, behavioral and neurodevelopmental disorders in the ICD-11: An international perspective on key changes and controversies. BMC Medicine 2020; 18:1-24

16. World Health Organization (WHO): International statistical classification of diseases and related health problems (11th ed.). 2019a. Available at: https://icd.who.int/en

17. World Health Organization (WHO): Implementation or transition guide, World Health Organization, Geneva, $2019 b$

Correspondence:

Prof. Wolfgang Gaebel, MD, PhD

WHO Collaborating Centre for Quality Assurance and Empowerment in Mental Health

LVR-Klinikum Düsseldorf, Department of Psychiatry, Medical Faculty, Heinrich-Heine-University

Bergische Landstr. 2, 40629 Düsseldorf, Germany

E-mail: wolfgang.gaebel@uni-duesseldorf.de 